\title{
Cognitive change in learning from text: Gesturing enhances the construction of the text mental model
}

\author{
Ilaria Cutica ${ }^{1}$ and Monica Bucciarelli ${ }^{2}$ \\ ${ }^{1}$ Department of Economy, Management and Quantitative Methods, University of Milan, Milan, Italy \\ ${ }^{2}$ Department of Psychology and Center for Cognitive Science, University of Turin, Turin, Italy
}

\begin{abstract}
The literature on co-speech gestures has revealed a facilitating effect of gestures on both the listener's discourse comprehension and memory, and the speaker's discourse production. Bucciarelli (2007) and Cutica and Bucciarelli (2008) advanced a mental model account for the cognitive change produced by gestures: Gestures, whether observed or produced, favour the construction of a mental model of the discourse they accompany. In this paper, we focus on gesturing while studying, assuming that gesturing while reading a text also favours the construction of a mental model of the text. In two experiments we invited adult participants to study two scientific texts and confirmed the predictions deriving from the assumption that gestures favour the construction of a mental model of the text: Gesturing while studying resulted in more correct recollections and text-based inferences (Experiment 1) and loss of verbatim recall (Experiment 2).
\end{abstract}

Keywords: Gestures; Learning; Mental models; Text comprehension.

It is well known that gestures have a facilitating effect on different aspects of speech comprehension and production. Studies on the enactment effect purport that gestures enhance memory for speech. The term enactment refers to the finding that "in memory tasks involving lists of action phrases like 'wave good-bye', 'put a glove', etc., recall and recognition scores are enhanced when the participants are instructed to perform the action during encoding (subject-performed task, SPT), compared to the standard condition in which they only read or listen to the phrases" (Feyereisen, 2009, p. 374). Some studies revealed that enactment is effective, although not in the same measure, also when the individual observes another person performing the action (experimenter-performed task, EPT) (e.g., Hornstein \&
Mulligan, 2004). More important, it has also been shown that the actual pattern of movements constituting a SPT is not crucial in determining the recall level, as long as the patterns are appropriate to the accompanying speech (e.g., Noice \& Noice, 2007). Noice and Noice (2007) detected the so-called nonliteral enactment effect, namely action-enhanced memory for verbal material that was not literally congruent with the performed actions, but was related to the movements at a higher order level (e.g., at the action goal level). Findings in the enactment literature are consistent with findings about co-speech gestures; this is not surprising, as SPTs and EPTs, especially when the gesture performed is not literally congruent with the speech, closely resemble co-speech gesture (e.g., Cohen, 1989).

Correspondence should be addressed to Ilaria Cutica, Department of Economy, Management and Quantitative Methods, University of Milan,Via Conservatorio, 7, 20133 Milan, Italy. E-mail: ilaria.cutica@unimi.it

This work was supported by Regione Piemonte, Italy, ATLAS project ID 44. 


\section{CUTICA AND BUCCIARELLI}

However, the studies on the enactment are mainly concerned with recognition and memory for lists of words or list of phrases (e.g., Feyer-esein, 2009), rather than discourses. As a conse-

quence, they disregard the beneficial effect of gestures on comprehension and learning in a broader view; in this respect, the studies on cospeech gestures are more relevant, as they also deal with the role of gestures in discourse comprehension.

The studies on co-speech gestures revealed that speaker's gesture facilitate hearer's discourse comprehension (see, e.g., Goldin-Meadow, 2000; Wagner Cook, Mitchell, \& Goldin-Meadow, 2008). Co-speech gestures provide symbolic and/ or analogic information that interacts with that conveyed by speech to reconstruct the communicative meaning by reinforcing, specifying or adding discourse contents.

To our knowledge, however, none of the studies in the literature on co-speech gestures have accounted for the cognitive mechanisms underlying their facilitating effect. We already advanced an account for the facilitating effects of co-speech gestures for the hearer (Bucciarelli, 2007; Cutica \& Bucciarelli, 2008, 2011): The information conveyed by the speaker's co-speech gestures, represented in a nondiscrete format, is easily included in the mental model of the discourse because mental models too are nondiscrete representations (see, e.g., Hildebrandt, Moratz, Rickheit, \& Sagerer, 1999).

As concerning the effect of gestures produced by the learner (in the learning phase), several studies found that concepts may be initiated in gesture before they are conveyed in speech (e.g., Wagner Cook et al., 2008), and that gestures may provide a vehicle that allows the individual to express thoughts difficult to express in speech (e.g., Goldin-Meadow, 2001). Indeed, gesticulation is also involved in the speaker's mental organisation of the discourse by helping to organise the stream of thought (see e.g., Alibali, Kita, \& Young, 2000), by reducing the cognitive load and leaving more resources available for other tasks (see, e.g., Alibali \& DiRusso, 1999), as well as by helping in the conceptual planning of utterances (see, e.g., Kita, 2000). Thus, gestures also have a facilitating effect for the speaker. In our terms, a speaker who has a good mental model of the concept to be expressed has less need to gesticulate than a speaker who has a poorer mental model (Cutica \& Bucciarelli, 2011).
The leading question of our investigation is: Does inviting a learner to gesticulate while study-ing a text have a beneficial effect on the con-struction of a complete mental model of the text content? This assumption is consistent with the results reported in the enactment literature sug-gesting that nonspontaneous gestures may also facilitate memory; it is, however, grounded on a unifying mental model framework, where ges-tures, whether observed or produced, facilitate the construction of a mental model of the text/ discourse.

\section{HOW GESTURING ENHANCES THE CONSTRUCTION OF A MENTAL MODEL OF THE TEXT}

The mental model theory may account for the cognitive changes brought about by gesturing while reading a text, and thus for the role of gesturing in the construction of the meaning of the text. Our main assumption is that gestures might lead to the construction of representations that are easily incorporated into the text model, alongside the representations constructed on the basis of the verbal information, enriching these and completing the mental model.

According with the literature, we considered as indicators of model construction the amount of correct recollections and of inferences produced by the learner, and the poor verbatim recognition of the text. Indeed, individuals who have built a good mental model of a given material are more likely to recall more correct information; and, more importantly, are more likely to draw correct inferences from the information explicitly con-tained in the material, with respect to individuals who, faced with the same material, have built a poorer mental model (Johnson-Laird, 1983). More specifically, we distinguish between dis-course-based inferences, based on mental models, and elaborative inferences, which embellish or add details to the text (see, e.g., Singer, 1994). Thus, elaborative inferences may interfere with the recovery of previously presented information, whereas discourse-based inferences are proper inferences. According to the search-aftermeaning theory, examples of discourse-based inferences are concerned with the causal antecedent, the causal consequent, the character's emotional reaction, and mental states (Graesser, Singer, \& Trabasso, 1994). 
Another possible indicator of the building of an articulated mental model of some given material is poor retention of the text or discourse surface form (for experimental evidence see, e.g., Garnham, Oakhill, \& Cain, 1998). This happens because mental models derived from a text or discourse do not encode the linguistic form of the sentences on which they are based. It follows that if the person constructs an articulated mental model of the information she/he has been ex-posed to, she/he will recognise less information at verbatim level with respect to a person who does not construct a mental model. As gestures favour the construction of mental models, which in turn lead to loss of verbatim recall, it follows that gestures do not favour the retention of the text/ discourse verbatim.

The findings within the enactment domain only apparently contrast with our expectation that gestures, by enhancing model construction, lead to loss of verbatim recall of the discourse. Indeed, within our proposed framework, a distinction is made between the effect of co-speech gestures on memory for sentences and on memory for dis-course. In the case of co-speech gestures accom-panying single sentences we assume that, in line with the enactment literature, gestures provide information that contributes to the creation of distinctive traces of the single sentences in the long-term memory. In the case of co-speech gestures accompanying connected sentences (i.e., discourse), we assume instead that cospeech gestures favour the construction of a mental model of the discourse. Multiple connected sentences give rise to the need to build an articulated discourse model. During this process, new pieces of information have to be integrated with one another and/or with prior knowledge, and any inconsistencies have to be solved. This is done by means of explanations, after which the discourse model that has been built so far has to be revised (see Khemlani \& Johnson-Laird, this issue).

Once a mental model of the discourse has been constructed, at the time of retrieval the representation of the information in the discourse is not in the form of distinctive traces; rather, the original information has been integrated into a single articulated mental model of the discourse. Hence, in order to tackle the construction of a mental model of the text, we invited the participants in our experiments to study a complete text. This gave them the possibility to build a single articulated mental model of the text and, as a consequence, draw discourse-based inferences and recognise less content at the verbatim level.

\section{EXPERIMENT 1: GESTURING ENHANCES LEARNING FROM TEXT (FREE RECALL TASK)}

The participants in the experiment were invited to study two scientific texts, one while gesticulating and the other without gesticulating. Then they were invited to recall as much information as they could. We expected to find that when people gesticulate while studying they (1) retain more information, and (2) draw more discourse-based inferences than when they do not gesticulate. Hence, we predicted a positive correlation be-tween number of gestures produced while study-ing and correct performances in the recall task. We had no predictions for the number of ela-borative inferences and errors. The former do not depend upon model construction, and errors are still possible when constructing a mental model of a text; any misunderstood information may be included in the mental model, thus supporting erroneous recollection.

\section{Method}

The experimental material consisted in two scientific texts, one concerning airplane flight (here-after, Airplane, see Appendix A1) and the other concerning the nature of sound (hereafter, Sound, see Appendix B1). We chose such scientific texts because we are interested in strategies that may aid learning, and scientific texts are a typical material that learners have to deal with. Further-more, the content of both texts is mostly concrete, and thus should be particularly apt to elicit gestures. Indeed, we already used both texts in a former study and we found that participants, when invited to explain them to a counterpart, were prone to accompany with gestures their explanations.

Each participant encountered both texts, one in the Gesture condition and the other in the No Gesture condition. Half of the participants dealt first with the gesture condition, and half with the no gesture condition; in each group, half of the participants encountered first the Airplane text, and half of them the Sound text. In the gesture condition participants were invited to gesticulate 
while studying the text: They were asked to represent the concepts they read in the text with gestures. Participants in the no gesture condition were invited to study the text while keeping their hands still. Participants in each experimental condition read each text twice, following the same experimental procedure. After completing the second reading of each text, participants were invited to recollect as much information as they could. All of the participants were videorecorded.

To code the results, each text was divided into 52 semantic units, corresponding to as many main concepts that the learner could recall. Each concept (i.e., semantic unit) recalled by the participants was evaluated by two independent judges according to the following coding schema:

-Literal recollection: a semantic unit recollected literally.

- Correct recollection: a semantic unit recollected through the participant's own words. ${ }^{\text {' }}$ - Discourse-based inference: a recollection in which the participant gave explicit information that was originally implicit in the semantic unit.

- Elaborative inference: a semantic unit recollected with the addition of plausible details.

- Erroneous recollection: a recollection with a meaning that was inconsistent with the semantic unit.

Two more judges examined the gestures produced by the participants while reading the texts. They defined hand movements that had a clear beginning and an end point, and that were also temporally linked to the reading of an utterance in the text as gestures. Then they counted the number of gestures produced by each participant in the experiment. None of the participants in the no gesture condition produced any gestures.

Participants. Forty adults took part in the experiment (27 females, 13 males, mean age: 22$)$; all were university students attending a

\footnotetext{
${ }^{1}$ Although a correct recollection may assume the form of a literal recollection, we consider as correct recollections only those in which participants reformulate through their own words the content in the semantic units. Indeed, in our framework, literal recollections are not apt to reveal the construction of a complete mental model of the text: It is possible to have a verbatim recollection even when the underlying mental model is incomplete or not fully integrated (see predictions of Experiment 2).
}

course in General Psychology, and all took part voluntarily.

\section{Results and discussion}

Two independent judges coded the participants' recollections individually; they reached a signifi-cant level of agreement on their first judgements (Cohen's $\mathrm{k}$ ranging from .80 to .89 , all psB.001). For the final score, the judges discussed each item on which they disagreed, until reaching a full agreement. Consider, for instance, the following semantic unit in the text: "Some of the air flows over the upper part of the wing". According to the coding schema, the statement "part of the air that hits the wing runs on the upper side of the wing" is a correct recollection, and the statement "the air hits the wing and passes underneath the wing" is an erroneous recollection. With respect to the semantic unit: "For an example where this can be seen (the perturbation in the environment begins to spread out, away from the source in all directions), think of waves on water", the sen-tence "An example is when an object falls into the water and produces concentric circles" is a discourse-based inference (it cites the cause of the effect that is mentioned). Finally, the sentence "How is it possible that an airplane can go up and fly for long distances?" is an elaborative inference of the semantic unit: "What makes an airplane rise into the sky and stay there even though it is heavier than air?" Table 1 shows the mean scores for types of recollection in the two experimental conditions.

Results show that the two texts were compar-able in difficulty: Considering each type of recol-lection separately, we found no differences in performance with the two texts: unpaired t-test, $t(38)$ comprised between 0 and $0.92, p$ comprised between .37 and 1 . Hence, we pooled together the results for the two texts. As a general result, and in both conditions, scores for correct recollections were higher than for other sorts of recollections. As predicted, there were more correct recollec-tions and discourse-based inferences in the gesture condition than in the no gesture condition: $t$-test for dependent samples, $t(39)$ ${ }_{3} 4.86$, tied $\mathrm{pB} .0001$ and $\mathrm{t}(39)_{3} 2.13$, tied $\mathrm{p}_{3} .02$, respectively. Literal recollections, elaborative inferences, and erro-neous recollections occurred to the same extent in the gesture condition and in the no gesture condition: t-test for dependent samples, $t(39)$ 
TABLE 1

Mean types of recollections in the gestures and no gestures conditions in Experiment 1

Condition (N 040)

Literal recollections

Correct recollections

Discourse-based inferences

Elaborative inferences

Errors
Gesture

M 0.05

No gesture

$\mathrm{M}$

SD
SD $\quad 0.22$

0.05
0.22

0.03
0.16

0.03
0.16
16.02

6.56

12.38

5.21
0.35
0.53
0.10
0.44

0.18

0.39

0.15

0.15
0.36
0.83

0.78

1.18

1.22 comprised between 0.17 and $0.14, \mathrm{t}(39)$ comprised between 0.17 and 0.77 , respectively.

Two more independent judges examined the gestures produced by each participant in the gesture condition and initially agreed to recognise $95 \%$ of their hand movements as gestures; they achieved a significant level of agreement (Cohen's $\mathrm{k}$ ranging from .82 to .89 , all psB.001). For the final score, the judges discussed each item on which they disagreed, until reaching a full agreement. By way of example, the following movement was considered to be an accompanying gesture; while reading the sentence "The upper surface of the wing is longer and more curved (than the lower surface)", the participant raised his right hand until it was level with his chest, holding it palm down and slightly concave, then drew an arc with his hand (moving it first upward then downward), outward with respect to the axis of the right-hand side of his body.

Table 2 shows the mean gestures produced by participants for each reading of each text.

Results show that participants in the gesture condition produced a comparable number of gestures while studying the Airplane and the Sound texts (over the two readings, a mean of 59.76 and 56.42 , respectively: unpaired t-test, $t(38) 00.55$, p 0.59. Hence, we pooled the results for the two texts. Also, participants produced a comparable amount of gestures in the first reading (a mean of 28.53 gestures) and in the second reading (a mean of 29.65 gestures): paired t-test, t(39) 00.86, p 0.40 .

TABLE 2

Mean of gestures performed for each reading of each text in Experiment 1

\begin{tabular}{ccccc} 
& \multicolumn{2}{c}{ Airplane } & \multicolumn{2}{c}{ Sound } \\
& $\begin{array}{c}\text { First } \\
\text { reading }\end{array}$ & $\begin{array}{c}\text { Second } \\
\text { reading }\end{array}$ & $\begin{array}{c}\text { First } \\
\text { reading }\end{array}$ & $\begin{array}{l}\text { Second } \\
\text { reading }\end{array}$ \\
M & 29.10 & 30.67 & 27.89 & \\
SD & 10.67 & 10.29 & 10.61 & 10.35
\end{tabular}

The total amount of gestures performed in both readings by each participant positively correlates with the amount of correct recollections, Pearson's r 0.51, pB.0001. No correlations were found among the amount of gestures and the other types of recollection, Pearson's $r$ comprised between .01 and .20, p comprised between .21 and .94.

Globally considered, the results of Experiment 1 confirmed the prediction that when people gesticulate while studying they retain more information, and draw more discourse-based inferences than when they do not gesticulate. Both sorts of recollection denote learning from text.

As we said earlier, a possible indicator of the building of an articulated mental model of a text is poor retention of the surface form. Hence, we predict that gesturing while studying should lead to a decrease in memory for text verbatim. This prediction is relevant in that one may argue that the effect of gesturing we observed in Experiment 1 was just a result of heightened arousal produced by gestures. If that would be the case, then gesturing would result in increased memory for text verbatim.

\section{EXPERIMENT 2: GESTURES IMPOVERISH VERBATIM RECOGNITION (RECOGNITION TASK)}

The participants in the experiment were invited to study two scientific texts, one while gesticulating and the other without gesticulating. Then they were presented with a list of sentences and were invited to say whether each sentence was present in the original text. We expected to find that when people gesticulate while studying they have worse verbatim recognition of the text than when they do not gesticulate, because a well-articulated mental model should lead to a good memory for contents at the expense of impoverished memory for surface information. 


\section{Method}

The experimental material consisted of the same two scientific texts used in Experiment 1. For each text we chose nine sentences, and for each sentence we created a triplet: (1) the very same sentence present in the text (literally correct); (2) a sentence with the same meaning, but expressed with different words (paraphrase); and (3) a sentence inconsistent in meaning (wrong content). We thus created 27 sentences, with nine in each category (literally correct, paraphrase, wrong content); see Appendix A2 and B2 for examples.

As in Experiment 1, each participant encountered both texts, one in the gesture condition and the other in the no gesture condition. Half of the participants dealt first with the Airplane text and half with the Sound text, and the occurrence of each text in the gesture and no gesture conditions was balanced across all participants. Participants in the gesture condition were invited to represent the concepts they read in the text with gestures. Participants in the no gesture condition were invited to study the text while keeping their hands still. The participants in each experimental condition read each text twice, following the same experimental procedure. As soon as the participants had finished studying each text, they were presented with the list of sentences, one by one in random order, and were asked to say whether or not the sentences were identical to those they had actually read in the text (recognition task). We coded responses of "Yes" to literally correct sentences, and responses of "No" to paraphrases2 and wrong content sentences as correct. (i.e., literally correct, paraphrase, wrong content), we found no differences in performance between the two texts: unpaired t-test, $\mathrm{t}(38)$ between 0.04 and 1.36, p between .19 and .94. Further, in order to verify our implicit assumption that participants would experience the same level of ease/difficulty in recognising the stimuli pertaining to each sentence category, we conducted several analyses of variance (Cochran's Q-test). The results revealed that the stimuli were comparable in difficulty in both the gesture $(Q$ value ranging from 14.23 to $19.41 ; p$-value ranging from .12 to $.42)$ and no gesture ( $Q$ value ranging from 11.79 to 22.12; $p$-value ranging from .10 to .48) conditions.

Table 3 illustrates the mean correct performance in the gesture and no gesture conditions.

As predicted, a series of t-tests for dependent samples revealed that participants performed worse in the gesture condition than in the no gesture condition in recognising sentences that were actually present in the original texts, $t(39) 0$ 3.14 , tied $p$ 0.003. Moreover, performance with paraphrases, t(39) 00.08, p 0.93, and wrong content sentences, t(39) 00.84, p 0.41, was comparable for participants in the two conditions.

\section{GENERAL DISCUSSION}

490

Previous studies in the literature have revealed that gestures facilitate both discourse comprehension and production. Our studies also revealed that gestures facilitate learning while studying a text, at least on all those occasions in which it is more important to grasp the meaning of the text rather than keep memory for text verbatim. We advanced a mental model account for the role of gestures in the construction of the meaning of a discourse/text, thus offering a unifying perspective on the role of gestures for the speaker and for the listener, as well as for the learner.
Participants. Forty students at Turin University (29 females, 11 males, mean age: 22), attending a course in General Psychology took part in the experiment. All took part on a voluntary basis and none had participated in Experiment 1.

\section{Results and discussion}

The two texts were comparable in difficulty: Considering each type of sentence separately

In Experiment 1, which involves a recollection task, the production of a paraphrase corresponds to a correct performance. In Experiment 2, which is a recognition task concerning text verbatim, the acceptance of a paraphrase of the original sentence in the text corresponds to an incorrect performance.
TABLE 3

Mean correct performance with the different sorts of sentences in the two experimental conditions in Experiment 2

\begin{tabular}{lccc}
\hline $\begin{array}{l}\text { Condition } \\
\text { (N 040) }\end{array}$ & $\begin{array}{c}\text { Literally correct } \\
(\mathrm{n} \mathrm{09})\end{array}$ & $\begin{array}{c}\text { Paraphrases } \\
\text { (n 09) }\end{array}$ & $\begin{array}{c}\text { Wrong content } \\
\text { (n 09) }\end{array}$ \\
\hline Gestures & & & \\
M & 6.10 & 5.05 & 5.80 \\
SD & 1.59 & 1.73 & 2.44 \\
No gestures & & & \\
M & 7.65 & 5.10 & 5.25 \\
SD & 1.53 & 2.10 & 1.65 \\
& & & \\
\hline
\end{tabular}


Is there any possible alternative account for the role of gestures in the cognitive change underlying learning from text/discourse? One of the most influential theories of discourse comprehension is the Construction Integration $(\mathrm{Cl})$ model of comprehension advanced by Kintsch (1998/2007), which is an extension of the theory formerly advanced by van Dijk and Kintsch (1983). According to this theory, text comprehension occurs through the construction of a situational model of the text contents. Disregarding their different theoretical roots, the terms "situational model" and "mental model" can be considered to be equivalent. However, in our view the concept of "mental model" can more easily accommodate the role of gestures in text/discourse comprehension. In the $\mathrm{Cl}$ model, Kintsch recognises the importance of incorporating extralinguistic knowledge in the modelling of discourse processing, but acknowledges that his model does not deal with this easily. In the $\mathrm{Cl}$ model, images, perceptions, concepts, ideas, or emotional states are translated into predicateÁargument units because of practical considerations (Kintsch, 1998/2007, p. 45): "We know how to work with predicateÁargument units, and it is not clear how to interface linear or spatial analog representations with such units." Kintsch claims that "[t]he predicateÁargument schema does not necessarily highlight relations that are significant in the realm of action and perception in a direct, analogous manner"' (p. 47). And, indeed, what he tries to grasp in his notation (network of nodes) is the analogical structure of mental models 'a la Johnson-Laird (pp. 108Á109).

Our assumptions on the role of gestures do not contrast with the Cl model by Kintsch (1998/ 2007), which, however, faces several problems for the implementation of mental models constructed from nonverbal information. We argue that the mental model theory is a better candidate for explaining the mechanisms underlying the role of gestures in discourse and text comprehension because of its emphasis on the nondiscrete nature of mental models; the theory allows us to envisage a role for co-speech gestures (which visually convey information in a nondiscrete, iconic representational format) within a theory of discourse/text comprehension. A further challenge for the $\mathrm{Cl}$ model is to account for the loss of verbatim recall following the construction of a mental model of the discourse/text; one further main difference between the mental model theory and the $\mathrm{Cl}$ model is that the former emphasises the fact that text verbatim is lost once the model

has been constructed; this prediction was verified by the results of our former and present studies.

In conclusion, the mental model theory appears to provide the most appropriate account for the cognitive change produced by gestures in discourse/text comprehension, production, and learning. Future studies might verify the effects of gestures on learning in studying subjects' texts other than scientific.

Original manuscript received May 2012 Revised manuscript received September 2012 First published online month/year

\section{REFERENCES}

Alibali, M. W., \& DiRusso, A. A. (1999). The function of gesture in learning to count: More than keeping track. Cognitive Development, 14, 37 Á56.

Alibali, M. W., Kita, S., \& Young, A. J. (2000). Gesture and the process of speech production: We think, therefore we gesture. Language and Cognitive Processes, 15(6), 593Á613.

Bucciarelli, M. (2007). How the construction of mental models improves learning. Mind and Society, 1(6), 67 89 .

Cohen, R. L. (1989). Memory for action events: The power of enactment. Educational Psychology Review, 1, 57Á80.

Cutica, I., \& Bucciarelli, M. (2008). The deep versus the shallow: Effects of co-speech gestures in learning from discourse. Cognitive Science, 32, 921Á935.

Cutica, I., \& Bucciarelli, M. (2011). "The more you gestured, the less I gesture": Co-speech gestures as a measure of mental model quality. Journal of Nonverbal Behavior, 35, 173Á187.

Feyereisen, P. (2009). Enactment effects and integration processes in younger and older adults' memory for actions. Memory, 17, 374Á385.

Garnham, A., Oakhill, J., \& Cain, K. (1998). Selective retention of information about the superficial form of text: Ellipses with antecedents in main and subordinate clauses. Quarterly Journal of Experimental Psychology, 51A(1), 19 Á39.

Goldin-Meadow, S. (2000). Beyond words: The importance of gesture to researchers and learners. Child AQ1 Development, 71, 231 Á139.

Goldin-Meadow, S. (2001). Giving the mind a hand: The role of gesture in cognitive change. In J. McClelland \& R. S. Siegler (Eds.), Mechanisms of cognitive development: Behavioral and neural perspectives (pp. 5Á31). Mahwah, NJ: Lawrence Erlbaum Associates, Inc.

Graesser, A. C., Singer, M., \& Trabasso, T. (1994). Constructing inferences during narrative text comprehension. Psychological Review, 101, 371 Á395.

Hildebrandt, B., Moratz, R., Rickheit, G., \& Sagerer, G. (1999). Cognitive modelling of vision and speech understanding. In G. Rickheit \& C. Habel (Eds.), Mental models in discourse processing and reasoning 
(pp. 213Á236). Amsterdam, The Netherlands: Elsevier/North Holland.

Hornstein, S. L., \& Mulligan, N. W. (2004). Memory for actions: Enactment and source memory. Psychonomic Bulletin and Review, 11, 367 Á372.

Johnson-Laird, P. N. (1983). Mental models: Towards a cognitive science of language and consciousness. Cambridge, UK: Cambridge University Press.

Kintsch, W. (2007). Comprehension: A paradigm for cognition. Cambridge, MA: Cambridge University Press. (Reprint, original work published 1998)

Kita, J. S. (Ed.). (2000). Pointing: Where language, culture, and cognition meet. Mahwah, NJ: Lawrence Erlbaum Associates, Inc.

Noice, H., \& Noice, T. (2007). The non-literal enactment effect: Filling in the blanks. Discourse Processes, 44, 73Á89.

Singer, M. (1994). Discourse inference processes. In M. A. Gernsbacher (Ed.), Handbook of psycholinguistics (pp. 479Á515). San Diego, CA: Academic Press.

van Dijk, I. A., \& Kintsch, W. (1983). Strategies of discourse comprehension. New York, NY: Academic Press.

Wagner Cook, S., Mitchell, Z., \& Goldin-Meadow, S. (2008). Gesturing makes learning last. Cognition, 106, 1047Á1058. again behind the wing./ The upper part of the wing is highly curved./ The air flowing over the upper part of the wing therefore/ has a greater distance to travel/ in the same period of time./ This means that the air flow over the top/ travels at a higher speed/ than that under the lower surface./ When air moves faster, its pressure reduces./ You will probably have noticed, when you turn on the water in the shower,/ that the curtain moves inwards./ The running water /makes the air inside the shower move faster./ At this higher speed, it exerts a lower pressure against the curtain/ than that exerted by the stationary air on the other side./ The curtain is thus forced inwards./ A similar principle applies to the aircraft wings./ Given that the air above the wing moves quicker,/ it tends to spread out./ The pressure on the upper part of the wing reduces./ The upper surface of the wing is at lower pressure/ than the lower surface of the wing./ The downward force exerted by the air flowing over the wing/ is less than the upward force from the air underneath,/ thus creating a net upward force on the wing ${ }^{\star}$ this is lift./ The lift generated by the wings must exceed the aircraft's weight./

A2: Examples of sentences used for the Recognition Task of Experiment 2

A1: The airplane flight text used in both Experiments 1 and 2 (semantic units are separated by slashes)

What makes an airplane rise into the sky and stay there, even though it is heavier than air?/ The answer lies/ in an aerodynamic principle/ suggested by Daniel Bernoulli/in 1738./ The Bernoulli principle describes how upward forces,/ known as lift,/ act on the aircraft as it moves through the air./ A transverse section of a bird's wing,/ a boomerang/ and a "Stealth" bomber/ all share a similar form to that of an airplane wing./ The upper surface of the wing is longer/ and more curved than the lower surface./ This difference creates what is known as an aerofoil./ To generate lift, air must flow over the wing./ A 747 has a wingspan/of over 200 feet,/ which is more than the height of a 15storey building./ As it moves around the wing,/ the air presses on it in all directions,/ at right angles to its surface./ When an airplane is in flight,/ the air divides as it hits the front of the wing./ Some of the air flows over the upper part of the wing,/ and the rest over the lower part of the wing./ The two air flows come together
Literal

wing.

Paraphrases During flight, the aircraft's wings split the air into two parts.

Wrong During flight, the air striking the aircraft's tail splits into two parts.

Literal When air moves faster, its pressure reduces.

Paraphrases As the air moves faster, the pressure that it exerts decreases.

Wrong Air moving very slowly is at lower pressure.

Literal The lift generated by the wings must exceed the aircraft's weight.

Paraphrases The aircraft must weigh less than the lift generated by its wings.

Wrong An aircraft's weight must be greater than the lift generated by its wings. 


\section{APPENDIX B}

B1: The nature of sound text used in both Experiments 1 and 2 (Semantic units are separated by slashes)

Physically, sound is a waveform:/ it consists of mechanical waves conveying energy/ away from the sound source,/ which is a vibrating object./ What travels, then, is not material but rather a signal,/ a continual vibration of some element/ of the environment in which the sound propagates./ It all starts with the vibration of an object/ or part of it,/ as with the tip of a tuning fork./ The perturbation in the environment begins to spread out /away from the source in all directions, / for an example where this can be seen think of waves on water./ This perturbation or signal makes every object vibrate that it meets on its path./ When the wave has passed by,/ everything returns to its original position./ There are two types of wave: longitudinal/and transverse./ With the former, the vibration occurs along the same axis/ as the wave's ravel;/ with the latter, the vibrations/ are at right angles to the wave's direction of travel./ An example of a longitudinal wave is the signal/ created by pressing on the end of a spring:/ the coils rhythmically/ move closer and further apart,/ while the signal propagates along the same axis on which the pressure was applied./ For a transverse wave, consider the signal/ created by a movement at one end of a rope:/ the signal propagates along the rope on an axis at right angles/ to the direction of the original movement./ Sound is a longitudinal wave,/ because the sound source vibrates in the same direction as the sound spreads./ This is what happens in a loudspeaker./ The waves may encounter many different objects as they spread out,/ but one of them is rather special: the eardrum./ This encounter triggers a highly intricate process/ that takes a sound signal/ and enables us to recognise a person's voice/ and understand the words they spoke,/ or to identify a musical instrument/ and even the musician playing it./ But this only explains part of what sound perception is all about./ Once our hearing apparatus has completed its work,/ we hear music,/ a deliberate and structured combination of sounds/ created to give aesthetic pleasure,/ language,/a deliberate and structured combination of sounds/ created to convey verbal information,/ noise,/ an ill-defined term used to refer to all other kinds of /unstructured, unpleasant, unwanted sounds./

B2: Examples of sentences used for the Recognition Task of Experiment 2

Literal : Physically, sound is a waveform.

Paraphrases: From a physics standpoint, sound is a wave.

Wrong : Physically, sound is sudden and irregular in nature.

Literal: It all starts with the vibration of an object.

Paraphrases: Sound is created by a vibrating object.

Wrong: Sounds are created by the body of a tuning fork.

Literal: This perturbation or signal makes every object vibrate that it meets on its path.

Paraphrases: Sound waves make every object vibrate that they meets on their path.

Wrong: Sound signals avoid the objects that they encounter on their path. 